\title{
Patient Care Outcomes of a Tobacco Use Registry in an Academic Family Practice
}

\author{
Carol Ripley-Moffitt, MDiv, Dana Neutze, MD, PhD, Mark Gwynne, DO, \\ and Adam O. Goldstein, MD, MPH
}

Purpose: While the potential benefit of a chronic disease registry for tobacco use is great, outcome reports have not been generated. We examined the effect of implementing a tobacco use registry, including a decision support tool, on treatment outcomes within an academic family medicine clinic.

Methods: A chart review of 200 patients who smoked and attended the clinic before and after registry implementation assessed the number of patients with clinic notes documenting (1) counseling for tobacco use, (2) recommendations for cessation medication, (3) a set quit date, (4) referrals to the onsite Nicotine Dependence Program (NDP) and/or QuitlineNC, and (5) pneumococcal vaccine. Data from the NDP, QuitlineNC, and clinic billing records before and after implementation compared the number of clinic-generated QuitlineNC fax referrals, new scheduled appointments for the NDP, and visits coded for tobacco counseling reimbursement.

Results: Significant increases in documentation occurred across most chart review variables. Significant increases in the number of clinic-generated fax referrals to QuitlineNC (from 27 to 96), initial scheduled appointments for the NDP (from 84 to 148), and coding for tobacco counseling (from 101 to 287) also occurred when compared with total patient visits during the same time periods. Patient attendance at the NDP (52\%) and acceptance of QuitlineNC services (31\%) remained constant.

Conclusions: The tobacco use registry's decision support tool increased evidenced-based tobacco use treatment (referrals, medications, and counseling) for patients at an academic family medicine clinic. This novel tool offers standardized care for all patients who use tobacco, ensuring improved access to effective tobacco use counseling and medication treatments. (J Am Board Fam Med 2015;28:205-213.)

Keywords: Chronic Disease, Registries, Smoking Cessation, Substance Abuse, Tobacco Use

Tobacco use remains the leading cause of preventable disease and death in the United States, and smoking accounts for nearly half a million prema-

This article was externally peer reviewed.

Submitted 18 April 2014; revised 8 October 2014; accepted 27 October 2014.

From the Department of Family Medicine, University of North Carolina School of Medicine, Chapel Hill.

Funding: none.

Prior presentation: This work was presented at the Society of Teachers of Family Medicine Practice Improvement Conference, Greenville, SC (2012); and the Society for Research on Nicotine and Tobacco Annual Meeting, Houston, TX (2012). Conflict of interest: none declared.

Corresponding author: Carol Ripley-Moffitt, MDiv, Department of Family Medicine, University of North Carolina School of Medicine, 590 Manning Drive, Chapel Hill, NC 27599-7595 (E-mail: carol_ripley-moffitt@med.unc.edu).

See Related Article on Page 214. ture deaths annually. ${ }^{1}$ Because tobacco abstinence reduces tobacco-related morbidity and mortality, treating tobacco use should be a key component of providing high-quality health care to patients. Research has demonstrated the critical importance of the physician's role in tobacco use treatment. ${ }^{2}$ Clinical practice guidelines recommend that tobacco use be addressed at each office visit with brief interventions using the "5 As" (ask about tobacco use; advise cessation; assess willingness to quit, assist in a quit attempt; and arrange follow-up), combined with offers of pharmacotherapy. ${ }^{3}$ Meta-analyses have found that brief interventions double-and the use of pharmacotherapy triples-a patient's chances of successfully quitting. ${ }^{3}$

Substantial progress has been made in tobacco use treatment interventions (eg, quit lines, medications, and feedback systems). Unfortunately, rates of counseling and offers of medication remain low. ${ }^{4}$ 
Data from the 2005 to 2009 National Ambulatory Medical Care Survey and National Health Interview Survey indicate that, among patients who use tobacco, only $20.9 \%$ received counseling and $7.6 \%$ received cessation medication. ${ }^{5}$ Physician time constraints and insufficient knowledge are among the most commonly cited barriers to providing tobacco use treatment to patients. ${ }^{6}$ Workflow-focused interventions can improve the implementation of recommendations with or without concurrent provider education interventions. ${ }^{7}$ One effective workflowfocused intervention, a clinical decision support tool, can increase guideline-oriented care for many chronic conditions. ${ }^{8}$ If decision support tools are automatically supplied to providers as part of clinician workflow, guideline implementation can improve $>75 \%$. ${ }^{9}$

Improvement in clinical outcomes and processes of care has also been seen in studies that include the use of disease registries for chronic disease management. ${ }^{10-12}$ With the adoption of quality improvement measures such as the electronic health record (EHR), identifying and tracking specific diseases and conditions within a clinic's patient caseload via a disease registry is becoming more feasible. While a meta-analysis of smoking cessation treatments found that multicomponent interventions, including screeners in the EHR, improve smoking outcomes, none specifically used a tobacco use registry. ${ }^{13} \mathrm{~A}$ tobacco use registry is thus a promising option to ensure that comprehensive structured care and coordinated counseling occur for patients who use tobacco.

The University of North Carolina Family Medicine Center (FMC) has for many years successfully used disease registries to support clinical decision making for patients with diabetes, congestive heart failure, and coronary artery disease. In the autumn of 2011 the FMC initiated a tobacco use registry, with the goal of standardizing evidence-based treatment for tobacco use. The health care system had already adopted several tobacco indicators as part of the vital sign section of the EHR, including current tobacco use, readiness to quit, and a reminder to advise those who use tobacco to quit. The design of the new tobacco registry significantly augmented the existing EHR by involving clinical medical assistants and providers in all phases of evidenced-based tobacco treatment, as well as vaccines and screenings recommended for this patient population. This article describes the outcomes of the FMC tobacco use registry, specifically those related to the decision support tool that was generated with each visit. To our knowledge, this is the first report of such a chronic disease decision support tool applied to tobacco use.

\section{Methods}

\section{Registry Design and Implementation}

Members of the FMC quality improvement team created a tobacco use registry modeled on the clinic's existing chronic disease registries and the decision support tools used at each patient visit. The tobacco use registry also aligned with the patientcentered medical home (PCMH) level 3 requirements of assessment and treatment of behavioral health conditions. Planning for the registry began four months before implementation. The November 2011 roll-out included only patients who were also part of the existing chronic disease registries; by mid-December 2011 it included all patients with a current tobacco use status during the past year.

The registry's decision support tool form was piloted with a team of providers (ie, resident and attending physicians, nurse practitioners) and medical assistants and went through 3 iterations based on feedback received. Once implemented, it was printed along with the other registry decision support tools each evening for the following day's patients (see Appendix). During patient visits, medical assistants used the tool to assess the current level of tobacco use and ask about quitting. If patients expressed readiness to quit, they were offered a referral to the onsite Nicotine Dependence Program (NDP) and to the North Carolina quit line (QuitlineNC), and they could choose to use one, both, or neither. If patients were not ready to quit, medical assistants gave patients a self-management tool to help identify triggers and motivators and provide resources. For male patients ages 65 to 75 , a box appeared to record the date of abdominal aortic aneurysm screening or was blank to remind medical assistants to recommend screening to patients. ${ }^{15}$ Medical assistants also were instructed to record the date of the most recent pneumococcal vaccine, which is recommended for patients older than 19 who currently smoke, as well as for all patients age 65 or older. ${ }^{16}$ Providers used the information obtained by the medical assistants and a list of prompts for recommended services (ie, fax referral to QuitlineNC, referral to NDP, offer medication, pro- 
vide self-management support, pneumococcal vaccine, 2-item Patient Health Questionnaire depression screen, aortic aneurysm screen) to guide their advice to patients and to develop an appropriate treatment plan that included counseling and pharmacotherapy. Once these actions were satisfied by checking boxes or adding dates on the part of the medical assistant and/or the provider, the recommended services were removed from the printed decision support tool at successive visits. ${ }^{17}$

\section{Chart Review}

The chart review included patients whose vital signs indicated current tobacco use for visits both before and after implementation of the registry. We obtained a list of all patients in the tobacco registry who attended the clinic from December 15, 2011, through March 31, 2012, and matched it with a list of patient visits in the year preceding registry implementation (November 1, 2010, through October 31, 2011). We selected the 3 -month time frame after implementation as an adequate time to evaluate the initial impact of the registry, as well as provide sufficient patient visits for randomization. We excluded pediatric patients $<18$ years of age. Of the 759 patients who attended the clinic in the 2 time periods, 200 were selected for study inclusion by choosing every third patient on the list. This represented over a quarter of the eligible population. Limited resources and time prohibited a chart review of all eligible patients.

In each patient chart in the EHR, we looked at the last visit before implementation of the tobacco use registry and the first visit after implementation. For both visits we assessed the following: counseling for cessation, cessation medications recommended and/or prescribed, establishment of a quit date, referral to QuitlineNC, referral to NDP, and evidence of new pneumococcal vaccination. These data reflect only the reports of providers through clinic notes and medication lists, except for the pneumococcal vaccine, which may have been entered into the EHR by medical assistants. While referral to the NDP and QuitlineNC may have been offered and checked on the registry form by the medical assistant, only the provider's note is counted in this analysis. The University of North Carolina Institutional Review Board approved the chart review study.

\section{Other Data Sources}

We used the following data sources to evaluate initial outcomes, looking at the 6 months before implementation (May-October 2011) and the first full 6 months after implementation (January-June 2012) to give sufficient time for comparing and analyzing trends.

\section{QuitlineNC Reports}

We used QuitlineNC's Fax Referral Report to validate the FMC count of clinic-generated fax referrals, using completed fax referrals as the final number. The report also gives the number of patients who accepted services.

\section{NDP Database}

While the chart audit documents referral, the NDP database shows the number of patients who were scheduled to attend and who actually attended the NDP clinic. It also records referral source; services are not limited to FMC patients.

\section{Billing Database}

Our clinic business administrator developed a monthly report from the health care system's Business Objects database. This included all visits to the FMC with 99406, 99407, G0436, or G0437 tobacco counseling codes billed. In addition, the administrator ran reports on the total number of patient visits to FMC providers during the time periods before and after registry implementation.

\section{Data Analysis}

We analyzed data from the chart audit with the McNemar test using STATA software version 12 (StataCorp, College Station, TX). We used the $Z$ test of proportions to compare the total number of QuitlineNC fax referrals, first-time NDP scheduled appointments, and use of prevention billing codes in the 6-month periods before and after the intervention with overall clinic visits. We used descriptive statistics to compare the rate of QuitlineNC services accepted, the number and percentage of patients scheduled at the NDP by referral source, and the rate of attendance at the NDP.

\section{Results \\ Chart Review}

Compared with all FMC patients who smoked, the study population was similar in race and sex but was 
Table 1. Demographics of Patients Represented in Chart Review $(n=200)$

\begin{tabular}{lc}
\hline Patient Demographics & \\
\hline Mean age, years (range) & $47(19-90)$ \\
Sex & \\
$\quad$ Female & $104(52)$ \\
Male & $96(48)$ \\
Race & \\
White & $126(63)$ \\
African American & $69(34.5)$ \\
Hispanic/other & $5(2.5)$ \\
Insurance & \\
$\quad$ Medicare & $33(16.5)$ \\
Medicaid & $61(30.5)$ \\
Private & $69(34.5)$ \\
No insurance & $37(18.5)$ \\
Chronic disease (diabetes, coronary heart disease, & $56(28)$ \\
$\quad$ and/or congestive heart failure) & \\
\hline
\end{tabular}

Data are $\mathrm{n}(\%)$ unless otherwise indicated.

slightly older (Table 1). A total of 70 of 78 primary care providers $(89.7 \%)$ are represented in the chart audit, of whom $47.1 \%$ were attending physicians, $44.3 \%$ were resident physicians, and $8.6 \%$ were nurse practitioners. Unique patients seen per provider averaged 4.3 (range, 1-18), with 98 patients $(49.0 \%)$ seeing the same provider in both time periods. Between both visits, 131 of the 200 patients $(65.5 \%)$ had at least 1 visit note documenting counseling for tobacco use.

The chart review showed that significant increases in counseling occurred, as documented by the provider in the clinic note. This was true for both attending and resident physicians $(P<.05)$. Prescription of cessation medications, the patient setting a quit date, and offers of referral to both the NDP and QuitlineNC also increased significantly (Figure 1). There was no significant difference in the percentage of patients who received pneumococcal vaccination.

\section{Otber Data Sources}

During the two 6-month time periods, the total number of visits to the FMC increased from 18,655 to $19,613(5.1 \%)$.

\section{Fax Referrals to QuitlineNC}

There was a significant increase in the number of clinic-generated fax referrals to QuitlineNC, from 27 in the 6 months before implementation of the
Figure 1. Percentage change in quality measures between the last visit before and the first visit after registry implementation $(\mathrm{n}=200) .{ }^{*} \boldsymbol{P}<.001, * * P<$ .05 , McNemar paired testing. NDP, Nicotine Dependence Program.

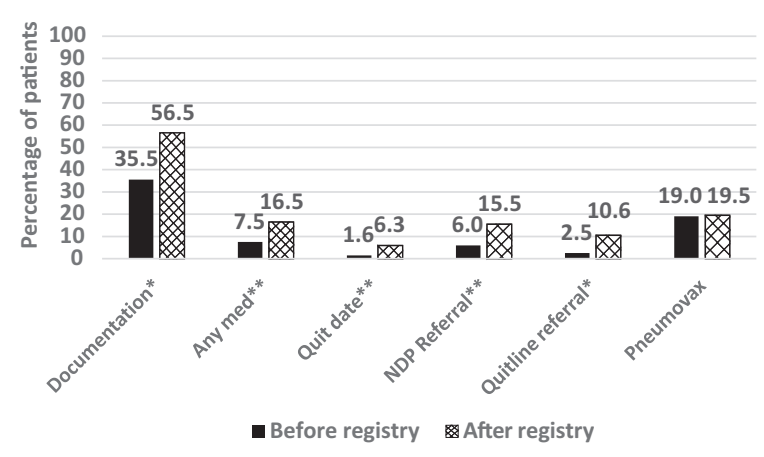

registry to 96 in the 6 months following implementation (Figure 2). Once the patient was contacted by the quit line, the acceptance rate for QuitlineNC services remained constant at $31.0 \%$ before and after implementation of the registry decision support tool.

\section{Patients Scheduled at the Specialty Clinic}

The number of scheduled first-time visits to the NDP increased significantly after implementation of the registry decision support tool, from 84 in the 6 months before registry implementation to 148 in the 6 months following implementation (Figure 2). These numbers include referrals by FMC providers, referrals by non-FMC providers, and patients who self-refer. However, the percentage of patients scheduled who were referred by FMC staff and providers increased from $57.6 \%$ to $75.3 \%$ in the

Figure 2. Number of fax referrals, appointments scheduled for the nicotine dependence program (NDP), and visits with billing codes for tobacco counseling 6 months before and 6 months after implementation of the tobacco use registry. $* P<.001$.

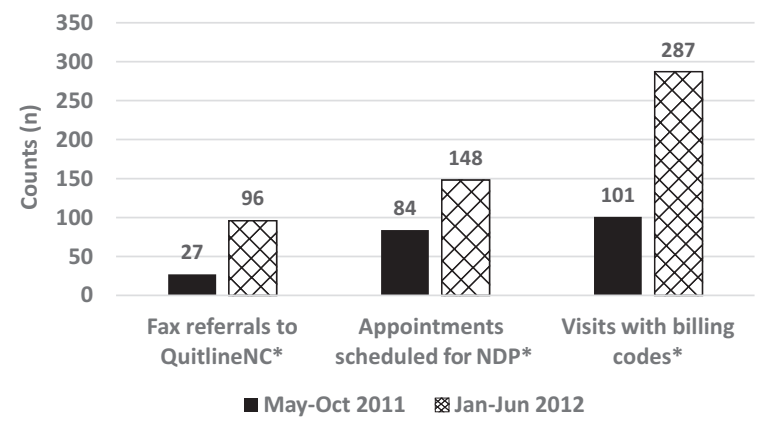


two 6-month time periods; referrals from nonFMC health care providers decreased from $40.0 \%$ to $10.9 \%$ of all scheduled visits, self-referral remained constant at $1.3 \%$, and those whose source of referral was unknown increased from $1.5 \%$ to $1.75 \%$. When compared with the total of all other referral sources, FMC referrals increased significantly $(P<.01)$. The percentage of patients who were scheduled and who actually attended NDP remained approximately $52 \%$ during the 2 time periods.

\section{Coding for Counseling}

Use of tobacco prevention counseling billing codes (for services longer than 3 minutes) significantly increased from 101 in the 6 months before the registry to 287 in the 6 months after implementation of the registry (Figure 2).

\section{Discussion}

To our knowledge, this study represents the first evaluation of a tobacco use registry used in a fashion similar to other chronic disease registries and reporting positive provider behaviors. Multiple corroborating pieces of evidence demonstrate that the use of the tobacco use registry decision support tool resulted in significant changes (a 2- to 4-fold increase) in the adoption of evidenced-based tobacco use treatments (counseling and pharmacotherapy). In many cases these increases are far beyond those achieved with other system-based interventions. ${ }^{18}$ While others have reported the impact of different delivery modes in the provision of the 5 As in the primary care setting, the studies have not included the application of a chronic disease registry or the use of broad measures associated with tobacco use, such as pneumococcal vaccine. ${ }^{13}$ Our tobacco registry form included depression screening prompts, which also were included in the other chronic disease registries. ${ }^{14} \mathrm{We}$ have not reported on this variable because we could not examine individual contributions from this registry.

Pharmacotherapy increases that occurred after implementation of our tobacco disease registry are particularly noteworthy; medication use in most studies is often $<10 \%$ and not consistent with recommendations. ${ }^{19}$ In the 6 months before registry implementation rates of medication offered to patients based on the chart review reflected the na- tional averages reported in the National Ambulatory Medical Care Survey. ${ }^{5}$ By doubling those rates in such a short time frame, our patients benefited from receiving a support that could double or triple their chances of quitting. ${ }^{3}$ We believe that the information in the tobacco use registry decision support tool on appropriate dosing and medication guidelines may have contributed to providers having easier access to and reminders about the 7 US Food and Drug Administration-approved cessation medications. ${ }^{17}$

The FMC tobacco use disease registry incorporated a team approach (eg, including medical assistants in the decision support tool process). Studies show that advice from multiple providers increases quit attempts. ${ }^{20}$ An approach that engages medical assistants in providing evidence-based care and initiating discussions that can be continued by providers make the patient-centered model more sustainable. This registry also fits the model of behavioral health interventions for substance use disorders: Screening, Brief Intervention, Referral to Treatment (SBIRT), which is similar to the 5 As. Integrating behavioral health into primary care is a hallmark of the PCMH. ${ }^{21}$ A tobacco use disease registry can standardize and help distribute selfmanagement materials so that all patients who use tobacco have good access to these resources. We noted that about a third of those receiving QuitlineNC fax referrals accepted services, and a little over half of those who were scheduled at the NDP actually attended. This is related to a conceptual model whereby the total number of patients receiving services increases by identifying those who have not received appropriate counseling and referral, even if the percentage following through does not change. $^{22}$

The increased coding for tobacco cessation counseling had a positive benefit on clinical revenue. The $161.0 \%$ increase in clinic revenue was a truly positive practice benefit. While this was not the primary incentive for providers to offer counseling, it does contribute to clinic operational costs. Both the clinic and individual providers received increased reimbursements for meeting $\mathrm{PCMH}$ and meaningful use goals (to which counseling for tobacco use contributes)- higher incentives than the prevention codes alone allowed.

There was no significant increase in pneumococcal vaccination rates. This may have been due to a lack of a standing order for the medical assistants 
to administer the vaccine. It may also not reflect true offers of the vaccine because we were unable to count in the chart audit patients who declined to receive the vaccine. We subsequently adopted a standing order for administration of the vaccine, and future evaluations will determine whether rates improve.

The question of sustainability, important in all quality improvement trials, was not addressed because we lacked funding to look at longer-term outcomes. We might expect that the rates of referral to the quit line and the onsite program might decrease because a majority of patients who smoke are reached during the year. A year after initiation, however, the use and return of registry forms continued to be high. ${ }^{17}$

\section{Limitations}

Several limitations to this data exist. First, a chart review does not capture the longitudinal nature of caring for a patient who uses tobacco. For example, interventions may have taken place during any of the visits other than the visits specifically reviewed for this study. While this may have skewed the data in either direction, it is unlikely to be a major contributor given that many of these patients were seen only twice during the time period studied. The applicability and generalizability of the results should take this into consideration. It may also be true that the documentation results do not reflect actual increased counseling, but rather that the registry triggered increased documentation. However, multiple process indicators and outcomes (eg, fax referrals, pharmacotherapy, referrals to cessation program) support the impact of the intervention.

This study did not report quit rates. Patient visits do not follow set time frames, for example, 3 , 6, 12 months, which would allow for consistent measurement. Moreover, the research on cessation strongly indicates that counseling and pharmacotherapy lead to increased quit attempts, increased quit rates, and sustained cessation. Finally, we focused on provider behavior and the provision of evidence-based care.

Costs of developing the registry database components were not factored into the outcomes, and these costs included hiring an outside information technology consultant, as well as increasing the workload of the administrative assistant who entered the data from the decision support tools into the chronic disease registry database on a daily basis. For smaller clinics, these costs might be prohibitive, although costs should be weighed against not only the reimbursement from actual counseling but also the increased revenue from meeting $\mathrm{PCMH}$ and meaningful use measures. The increased use of EHRs with functionality that incorporates aspects of this tobacco disease registry may require less initial start-up costs (ie, information technology support). Our work should also help to inform data fields to include in new EHRs, as well as options for reports. Finally, the sampling size was too small to determine whether changes in rates of abdominal aortic aneurysm screening occurred.

\section{Future Studies}

Futures studies could examine the cost versus the benefits of a tobacco use registry, the feasibility of using this tool or a similar one in a nonacademic clinic setting, how a patient care registry can also serve as a longitudinal database for quitting outcomes, and whether this type of registry can provide a vehicle for enrolling patients in clinical trials or research studies. We plan to use information from the registry database to communicate with patients in need of pneumococcal vaccination. We also are looking at adding to our registry forms the recent recommendations for computed tomography lung cancer screening by the US Preventive Services Task Force.

The authors thank Todd Meath, Kevin Tate, and James Garner for data collection and Jennifer Greyber for editorial assistance. In addition, the authors thank the Health-e-NC initiative of the University Cancer Research Fund at the University of North Carolina for financial support for manuscript preparation.

\section{References}

1. Mokdad AH, Marks JS, Stroup DF, Gerberding JL. Correction: Actual causes of death in the United States, 2000. JAMA 2005;293:293-4.

2. Stead LF, Buitrago D, Preciado N, Sanchez G, Hartmann-Boyce J, Lancaster T. Physician advice for smoking cessation. Cochrane Database Syst Rev 2013;5:CD000165.

3. 2008 PHS Guideline Update Panel, Liaisons, and Staff. Treating tobacco use and dependence: 2008 update U.S. public health service clinical practice guideline executive summary. Respir Care 2008;53: 1217-22.

4. 2014 Definition stage 1 of meaningful use. Baltimore (MD): Centers for Medicare and Medicaid Services. 
Modified October 6, 2014. Available from: http:// www.cms.gov/Regulations-and-Guidance/Legislation/ EHRIncentivePrograms/Meaningful_Use.html. Accessed January 12, 2015.

5. Jamal A, Dube SR, Malarcher AM, Shaw L, Engstrom MC; Centers for Disease Control and Prevention (CDC). Tobacco use screening and counseling during physician office visits among adults-National Ambulatory Medical Care Survey and National Health Interview Survey, United States, 2005-2009. MMWR Morb Mortal Wkly Rep 2012;61(Suppl): 38-45.

6. Berlin I. Physicians' perceived barriers to promoting smoking cessation. J Smok Cessat 2008;3:92-100.

7. Flanagan ME, Ramanujam R, Doebbeling BN. The effect of provider- and workflow-focused strategies for guideline implementation on provider acceptance. Implement Sci 2009;4:71.

8. Garg AX, Adhikari NK, McDonald H, et al. Effects of computerized clinical decision support systems on practitioner performance and patient outcomes: a systematic review. JAMA 2005;293:1223-38.

9. Kawamoto K, Houlihan CA, Balas EA, Lobach DF. Improving clinical practice using clinical decision support systems: a systematic review of trials to identify features critical to success. BMJ. 2005;330:765.

10. Tsai AC, Morton SC, Mangione CM, Keeler EB. A meta-analysis of interventions to improve care for chronic illnesses. Am J Manag Care 2005;11: 478-88.

11. Halladay JR, DeWalt DA, Wise A, et al. More extensive implementation of the chronic care model is associated with better lipid control in diabetes. J Am Board Fam Med 2014;27:34-41.

12. Chin MH, Cook S, Drum ML, et al. Improving diabetes care in midwest community health centers with the health disparities collaborative. Diabetes Care 2004;27:2-8.

13. Papadakis S, McDonald P, Mullen KA, Reid R, Skulsky K, Pipe A. Strategies to increase the delivery of smoking cessation treatments in primary care set- tings: a systematic review and meta-analysis. Prev Med 2010;51:199-213.

14. Arroll B, Goodyear-Smith F, Crengle S, et al. Validation of PHQ-2 and PHQ-9 to screen for major depression in the primary care population. Ann Fam Med 2010;8:348-53.

15. LeFevre ML. Screening for Abdominal aortic aneurysm: U.S. Preventive Services Task Force recommendation statement. Ann Intern Med 2014;161: 281-90.

16. Centers for Disease Control and Prevention (CDC); Advisory Committee on Immunization Practices. Updated recommendations for prevention of invasive pneumococcal disease among adults using the 23-valent pneumococcal polysaccharide vaccine (PPSV23). MMWR Morb Mortal Wkly Rep 2010; 59:1102-6.

17. Neutze D, Ripley-Moffitt C, Gwynne M, Goldstein AO. The implementation of a tobacco use registry in an academic family practice. J Am Board Fam Med 2014;28:214-21.

18. Bentz CJ, Bayley KB, Bonin KE, et al. Provider feedback to improve 5A's tobacco cessation in primary care: a cluster randomized clinical trial. Nicotine Tob Res. 2007;9:341-9.

19. Linder JA, Rigotti NA, Schneider LI, Kelley JH, Brawarsky P, Haas JS. An electronic health recordbased intervention to improve tobacco treatment in primary care: a cluster-randomized controlled trial. Arch Intern Med 2009;169:781-7.

20. An LC, Foldes SS, Alesci NL, et al. The impact of smoking-cessation intervention by multiple health professionals. Am J Prev Med 2008;34:54-60.

21. Fact sheet: patient-centered medical homes. Washington, DC: National Committee for Quality Assurance; 2011. Available from: http://www.ncqa.org/ portals/0/Public\%20Policy/PCMH_2011_fact_sheet_ 2.9.13.pdf. Accessed January 12, 2015.

22. Cohen D, McDaniel RR Jr, Crabtree BF, et al. A practice change model for quality improvement in primary care practice. J Healthc Manag 2003;49: 155-68; discussion 169-70. 
Appendix

Registry Decision Support Tool (Front and Back)

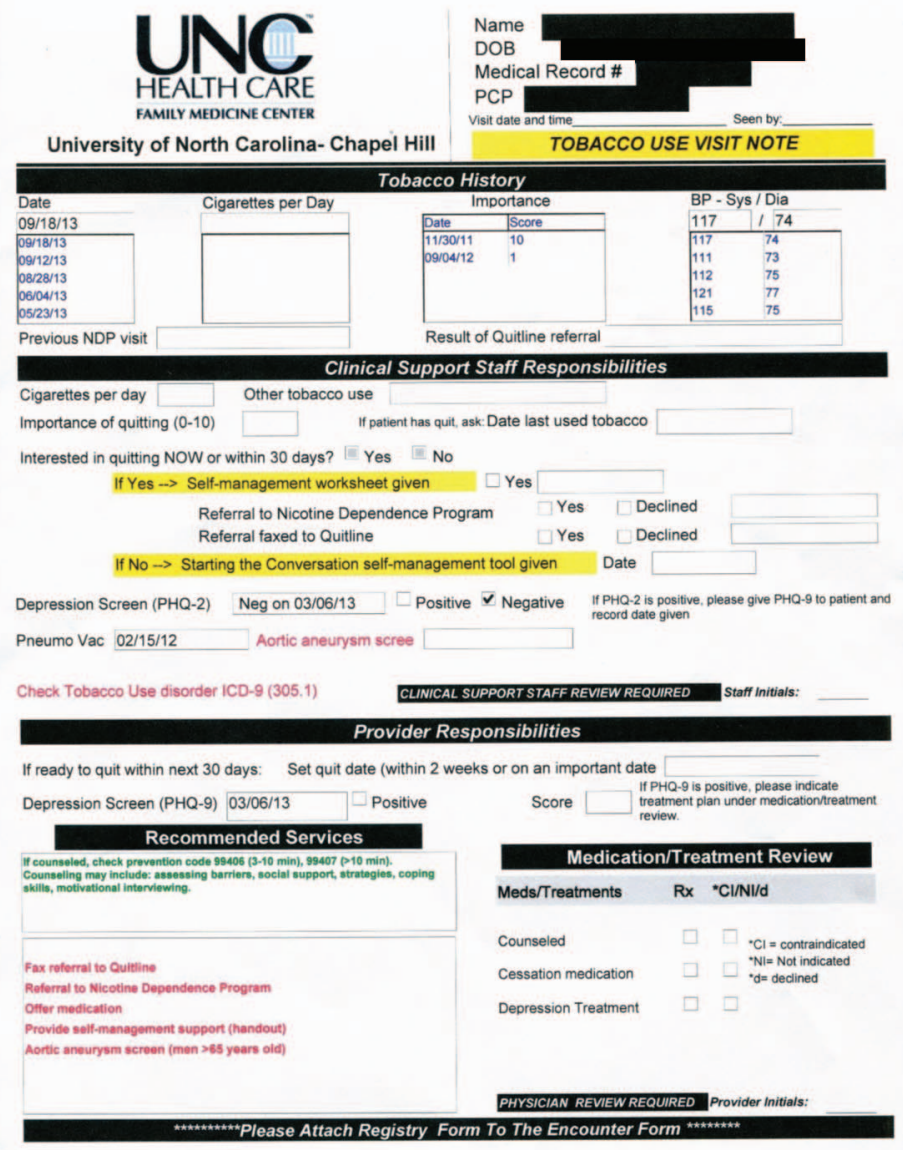

212 JABFM March-April 2015 Vol. 28 No. 2 
